\title{
DISTAL HOMOMORPHISMS OF NONMETRIC MINIMAL FLOWS
}

\author{
D. MMMAHON AND T. S. WU
}

\begin{abstract}
In a recent paper, $R$. Ellis proved that the Furstenberg structure theorem for distal minimal flows with metric phase space held for flows with nonmetric phase space. In this paper the authors remove the metric constraint from the relativized Ellis-Furstenberg structure theorem for distal homomorphisms and for the Veech structure theorem for point-distal minimal flows.
\end{abstract}

One of the most celebrated theorems in topological dynamics is Furstenberg's structure theorem for metric distal minimal flows: Let $(X, T)$ be a distal minimal transformation group with compact metric space $X$, then $(X, T)$ is the inverse limit of a family of distal minimal flows $\left(X_{n}, T\right)$ such that $\left(X_{n+1}, T\right)$ is an isometric extension of $\left(X_{n}, T\right)$. Motivated by this theorem, one of the main interests in topological dynamics has been to express the structure of a minimal set as an inverse limit of a family of minimal sets such that each extension is either distal or proximal or weakly mixing [V]. This approach has been very fruitful and much progress has been made in the last decade on the structure of metric minimal flows. However one of the most fundamental questions remained unanswered until recently: Can one drop the requirement on the metrizability of the phase space in Furstenberg's structure theorem? In 1978, R. Ellis used an ingenious method to provide a positive answer to the above. The basic idea of Ellis is that: Let $(X, T)$ be a distal minimal flow. If $(X, T)$ cannot be expressed as an inverse limit of isometric extensions of minimal sets, then there exists a countable subgroup $H$ of $T$ and a compact metric space $Y$ such that $(Y, H)$ has the same property and is a homomorphic image of $(X, H)$. But $(X, H)$ is distal and therefore $(Y, H)$ is distal, and we have a contradiction. thus Furstenberg's structure theorem holds for nonmetric spaces as well. At that time, McMahon and Nachman were investigating the structure of PI-flows and found an intrinsic characterization of such flows. Combining this characterization with Ellis's technique, they prove the nonmetric version of Veech's structure theorem (which is a generalization of Furstenberg's structure theorem to point-distal metric minimal flows).

In this paper we prove a relativized, nonmetric version of Furstenberg's theorem for distal homomorphisms of (possibly nonmetric) minimal flows. We also give an easier proof of the nonmetric version of Veech's structure theorem. We have been unable to prove a relativized nonmetric version of Veech's structure theorem.

Our proof stems from the following basic construction. Given $\theta:(X, T) \rightarrow$ $(Y, T)$ there is a countable subgroup $K$ of $T$ and metric minimal flows $X_{K}, Y_{K}$ and

Received by the editors March 24, 1980 and, in revised form, August 27, 1980.

1980 Mathematics Subject Classification. Primary 54H20. 
$K$-equivalent maps $\theta_{K}, \phi_{K}, \psi_{K}$ such that the diagram

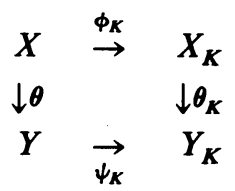

commutes, $\phi_{K}\left(\theta^{-1}(y)\right)=\theta_{K}^{-1}\left(\psi_{K}(y)\right)$, and $\phi_{K} \times \phi_{K}(Q(\theta)) \subseteq Q\left(\theta_{K}\right)$.

Note Theorem 1 and Corollary 2 are concerned with $Q, S$, distal points, and closed invariant equivalence relations (and thus homomorphisms), none of which depend on the topology on $T$, so we may assume without loss of generality that $T$ has the discrete topology; in Proposition 3 we assume that $T$ has the discrete topology.

Definitions and notations. In this paper we will assume that a flow $(X, T)$ has a compact Hausdorff phase space $X$ acted on the right by a topological group $T$ (often the acting group will be understood and we will write just $X$ in place of $(X, T))$. A continuous mapping $\phi$ of a flow $(X, T)$ onto a flow $(Y, K)$ where $K \subseteq T$ is called $K$-equivariant if $\phi(x k)=\phi(x) k$ for all $k$ in $K$ (when $K=T, \phi$ is a homomorphism). We denote the Stone-Čech compactification of $T$ by $\beta T$, a fixed minimal right ideal in $\beta T$ by $M_{T}$, and the set of idempotents in $M_{T}$ by $J_{T}$.

We denote the set of closed subsets of $X$ by $2^{X}$ and give it the Hausdorff topology, a basic neighborhood of $E$ in $2^{X}$ is $\{A: E \subseteq \alpha A$ and $A \subseteq \alpha E\}$ where $\alpha$ is a uniformity on $X$. With this topology $2^{X}$ is a compact Hausdorff space (see p. 113 of $\left[\mathbf{G}_{\mathbf{B}}\right]$ or see exercises, Chapter II, $\S 1,5(\mathrm{a})$; Chapter IX, $\$ 2,6(\mathrm{~b})$; and Chapter II, $\S 4,11$ of [B]). Now given any pseudometric, $d$, compatible with the topology on $X$ (i.e. $B(x, d, \varepsilon)=\{y: d(x, y)<\varepsilon\}$ is open in $X$ ) for each $\varepsilon>0$, we can form a uniformity on $X$ by $\alpha_{\varepsilon}=\{(x, y): d(x, y)<\varepsilon\}$ and the corresponding neighborhood of $E$ in $2^{X}$ is $V=\{A: E \subseteq\{x: d(a, x)<\varepsilon$ for some $a$ in $A\}$ and $A \subseteq\{x: d(e, x)$ $<\varepsilon$ for some $e$ in $E\}\}$.

Define $d^{*}$ on $2^{X}$ by

$$
d^{*}(A, E)=\max \left\{\max _{a \in A}\left[\min _{e \in E}(d(a, e))\right], \max _{e \in E}\left[\min _{a \in A}(d(a, e))\right]\right\} .
$$

Note the maximum and minimum exist since $A$ and $E$ are compact. Then one can verify $V=\left\{A: d^{*}(A, E)<\varepsilon\right\}$ and that $d^{*}$ is a pseudometric compatible with the topology on $2^{X}$.

We denote the proximal relation for a flow $(X, T)$ by $P(X, T), P_{X}$ or $P$. If $\theta: X \rightarrow Y$ is a homomorphism $R(\theta)=\left\{\left(x, x^{\prime}\right): \theta(x)=\theta\left(x^{\prime}\right)\right\}$ and $Q(\theta)$ denotes the relativized regional proximal relation, $Q(\theta)=\left\{\left(x, x^{\prime}\right)\right.$ : there exist nets $\left(x_{n}, x_{n}^{\prime}\right)$ in $R(\theta)$ and $t_{n}$ in $T$ with $\left(x_{n}, x_{n}^{\prime}\right) \rightarrow\left(x, x^{\prime}\right)$ and $\left.\left(x_{n}, x_{n}^{\prime}\right) t_{n} \rightarrow(x, x)\right\}$.

The relativized equicontinuous structure relation $S(\theta)$ is the smallest closed invariant equivalence relation such that the induced homomorphism $X / S(\theta) \rightarrow Y$ is almost periodic.

Basic construction. Suppose $\theta:(X, T) \rightarrow(Y, T)$ is an open homomorphism between minimal flows $X$ and $Y$. For any countable subgroup $K=\left\{k_{i}\right\}_{i-1}^{\infty}$ and 
continuous pseudometric $d$ on $X$ with $\max \left(d\left(x, x^{\prime}\right)\right)<1$, we define $d_{K}$ on $X \times X$ by $d_{K}\left(x, x^{\prime}\right)=\sum_{i=1}^{\infty} 2^{-i} d\left(x k_{i}, y k_{i}\right)$, and define closed $K$-invariant equivalence relations $R(X, K, d)$ and $R(Y, K, d)$ on $X$ and $Y$ respectively by

$$
R(X, K, d)=\left\{\left(x, x^{\prime}\right): d_{K}\left(x, x^{\prime}\right)=0\right\}
$$

and

$$
R(Y, K, d)=\left\{\left(y, y^{\prime}\right): d_{K}^{*}\left(y, y^{\prime}\right)=0\right\},
$$

where $d_{K}^{*}$ is the pseudometric induced on $2^{X}$ by $d_{K}$ and $(Y, T)$ is identified with a subset of $\left(2^{X}, T\right)$ by the homomorphism $y \rightarrow \theta^{-1}(y)$.

Note $d_{K}^{*}\left(y, y^{\prime}\right)=0$ means $d_{K}^{*}\left(\theta^{-1}(y), \theta^{-1}\left(y^{\prime}\right)\right)=0$ and so for each $x$ in $\theta^{-1}(y)$ there exists an $x^{\prime}$ in $\theta^{-1}\left(y^{\prime}\right)$ such that $d_{K}\left(x, x^{\prime}\right)=0$. Let $A_{K}=X / R(X, K, d)$ and $Y_{K}=Y / R(Y, K, d)$; let $\alpha_{K}: X \rightarrow A_{K}$ and $\psi_{K}: Y \rightarrow Y_{K}$ be the induced maps; and define $\phi_{K}: X \rightarrow A_{K} \times Y_{K}$ by $\phi_{K}(x)=\left(\alpha_{K}(x), \psi_{K}(\theta(x))\right)$. Let $X_{K}=\phi_{K}(X)$. Note $A_{K}$, $Y_{K}$ and $X_{K}$ are metric (e.g. the metric on $A_{K}$ is given by $d_{A}\left(a, a^{\prime}\right)=d_{K}\left(x, x^{\prime}\right)$ where $\alpha(x)=a$ and $\alpha\left(x^{\prime}\right)=a^{\prime}$, and is compatible with the quotient topology. Define $\theta_{K}: X_{K} \rightarrow Y_{K}$ by $(a, y) \rightarrow y$. Note $\alpha_{K}, \psi_{K}, \phi_{K}$, and $\theta_{K}$ are $K$-equivariant. We wish to show a correspondence between the fibers of $\theta$ and $\theta_{K}$, that

$$
\theta_{K}^{-1}\left(\psi_{K}(y)\right)=\phi_{K}\left(\theta^{-1}(y)\right) \text {. }
$$

Since the diagram

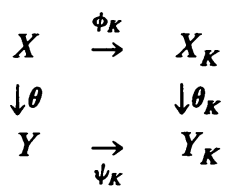

commutes, $\theta_{K}^{-1}\left(\psi_{K}(y)\right) \supseteq \phi_{K}\left(\theta^{-1}(y)\right)$. We wish to show $\theta_{K}^{-1}\left(\psi_{K}(y)\right) \subseteq \phi_{K}\left(\theta^{-1}(y)\right)$. To see what this entails note that

$$
\begin{aligned}
\theta_{K}^{-1}\left(\psi_{K}(y)\right) & =\left\{\left(\alpha_{K}(x), \psi_{K}(\theta(x))\right): \psi_{K}(\theta(x))=\psi_{K}(y), x \in X\right\} \\
& =\left\{\left(\alpha_{K}(x), \psi_{K}(y)\right): d_{K}^{*}(\theta(x), y)=0\right\} .
\end{aligned}
$$

So we wish to show that if $x \in X$ with $\psi_{K}(\theta(x))=\psi_{K}(y)$, then there eixsts an $x^{\prime}$ in $\theta^{-1}(y)$ with $\alpha_{K}\left(x^{\prime}\right)=\alpha_{K}(x)$, i.e., with $d_{K}\left(x, x^{\prime}\right)=0$. But this is clear since $0=$ $d_{K}^{*}(\theta(x), y)=d_{K}^{*}\left(\theta^{-1}(\theta(x)), \theta^{-1}(y)\right)$. Thus the fibers of $\theta_{K}$ are images of fibers of $\theta$. Note also $\left(X_{K}, K\right)$ and $\left(Y_{K}, K\right)$ are flows.

We will now use this relationship between fibers to show that $\theta_{K}$ is open. Suppose $\theta_{K}$ is not open at $x^{*}$ in $X_{K}$. Then for some (open) neighborhood $V$ of $x^{*}$ the set $\theta_{K}(V)$ does not contain a neighborhood of $y^{*}=\theta_{K}\left(x^{*}\right)$. Let $y_{n}^{*}$ be a net in $Y_{K}$ converging to $y^{*}$ with $y_{n}^{*} \notin \theta_{K}(V)$. Let $y_{n} \in \psi_{K}^{-1}\left(y_{n}^{*}\right)$ and $y_{n_{1}}$ be a convergent subnet of $y_{n}$; let $y$ be its limit. Then $\psi_{K}(y)=y^{*}$ and since $\theta_{K}^{-1}\left(y^{*}\right)=\phi_{K}\left(\theta^{-1}(y)\right)$ there is an $x$ in $\theta^{-1}(y) \cap \phi_{K}^{-1}\left(x^{*}\right)$. Then $\phi_{K}^{-1}(V)$ is a neighborhood of $x$ and $\theta\left(\phi_{K}^{-1}(V)\right)$ is a neighborhood of $y$ since $\theta$ is open. So $y_{m} \in \theta\left(\phi_{K}^{-1}(V)\right)$ for some $m$ and for some $x^{\prime} \in \theta^{-1}\left(y_{m}\right), x^{\prime} \in \phi_{K}^{-1}(V)$. Then $\phi_{K}\left(x^{\prime}\right) \in V$ and $\phi_{K}(V) \ni \theta_{K}\left(\phi_{K}\left(x^{\prime}\right)\right)$ $=\psi_{K} \theta\left(x^{\prime}\right)=\psi_{K}\left(y_{m}\right)=y_{m}^{*} \notin \theta_{K}(V)$; a contradiction. Thus $\theta_{K}$ is open.

Fix some $x_{0}$ in $X$; we will construct $K$ so that $\phi_{K}\left(x_{0}\right)$ is an almost periodic point and has dense orbit $\phi_{K}\left(x_{0}\right) K$, and $\phi_{K} \times \phi_{K}(Q(\theta)) \subseteq Q\left(\phi_{K}\right)$. We will do so by 
constructing a sequence of subgroups $K_{i}$ of $T$ with $K_{i} \subseteq K_{i+1}$ and taking $K=$ $\cup K_{i}$. We note that $X_{K}=\operatorname{inv} \lim X_{K_{i}}, Y_{K}=\operatorname{inv} \lim Y_{K_{i}}$, and $\phi_{K}, \psi_{K}, \theta_{K}$ are identical to the maps obtained via the inverse limit from $\phi_{K_{i}}, \psi_{K_{i}}, \theta_{K_{i}}$. Also recall that in an inverse limit, $W=$ inv $\lim _{i \in I} W_{i}$, a basic open set on $W$ is of the form $\gamma_{i}^{-1}(V)$ for some $i$ and some basic open set $V$ in $W_{i}$ where $\gamma_{i}: W \rightarrow W_{i}$. Let $B_{i}$ be a countable basis for $X_{K_{i}}$. Denote $X_{K_{i}}, Y_{K_{i}}$, etc. by $X_{i}, Y_{i}, \ldots$

Now to construct $K$, start with $K_{1}=\{e\}$ where $e$ is the identity. Given $K_{i}$, we construct $K_{i+1}$ by adding some elements of $T$ to $K_{i}$ and generating the subgroup containing them. In order for $\phi_{K}\left(x_{0}\right)$ to have dense orbit $\phi_{K}\left(x_{0}\right) K$ in $X_{K}$, we add for each $V$ in $B_{i}$ some $t$ in $T$ such that $x_{0} t \in \phi_{i}^{-1} k(V)$. In order for $\phi_{K}\left(x_{0}\right)$ to be an almost periodic point we add for each $V$ in $B_{i}$ some finite subset $F$ of $T$ such that $X \subseteq \phi_{i}^{-1}(V) F$. In order for $\phi_{K}(Q(\theta)) \subseteq Q\left(\theta_{K}\right)$ to hold we add, for each $V, W$ in $B_{i}$ with $\left[\phi_{i}^{-1}(V) \times \phi_{i}^{-1}(W)\right] \cap Q(\theta) \neq \varnothing$, some $t$ in $T$ such that for some $\left(x, x^{\prime}\right)$ in $\left[\phi_{i}^{-1}(V) \times \phi_{i}^{-1}(W)\right] \cap R(\theta)$, we have $\left(x t, x^{\prime} t\right) \in B\left(d_{i}, x_{0}, 1 / n\right) \times B\left(d_{i}, x_{0}, 1 / n\right)$.

Forming $K=\cup K_{i}$ we see that $\phi_{K}\left(x_{0}\right)$ is an almost periodic point in $\left(X_{K}, K\right)$ with dense orbit and $\phi_{K}(Q(\theta)) \subseteq Q\left(\theta_{K}\right)$.

We need some more observations before proving our main theorem.

(i) Suppose $\theta$ is not proximal; let $y_{0}=\theta\left(x_{0}\right), X_{0}=\theta^{-1}\left(y_{0}\right)$, and $x_{1} \in X_{0}$ with $\left(x_{1}, x_{0}\right) \notin P_{X}$. Then $\operatorname{cls}\left(\left(x_{0}, x_{1}\right) T\right) \cap \Delta=\varnothing$ where $\Delta$ is the diagonal, $\Delta=\{(x, x): x$ $\in X\}$. Take a pseudometric $d$ on $X$ such that $d\left(x, x^{\prime}\right)=1$ for all $\left(x, x^{\prime}\right) \in$ $\operatorname{cls}\left(\left(x_{0}, x_{1}\right) T\right)$ and $\operatorname{diam}(X)=1$. We have $\left(\phi_{K}\left(x_{1}\right), \phi_{K}\left(x_{0}\right)\right) \in P_{X_{K}}$ and $\theta_{K}$ is not proximal. In particular, no fiber of $\theta_{K}$ is a singleton.

(ii) Consider any subgroup $K$ of $T$. Let $u_{K} \in J_{K}$ and $u_{T} \in J_{T}$. Then for some $u \in J_{T}, u_{T} u_{K} u=u_{T} u_{K}$ and there exists $p \in M$ such that $p\left(u_{T} u_{K}\right)=u$. So $u_{T} u_{K} u u_{K}$ $=u_{T} u_{K} u_{K}=u_{T} u_{K}, p u_{T} u_{K} u u_{K}=p u_{T} u_{K}$, and thus $u u_{K}=u$. That is, for any $u_{K} \in J_{K}$ there exists $u \in J_{T}$ with $u u_{K}=u$.

(iii) Finally note by 2.6 .2 of [V], when the almost periodic points in $R(\theta)$ are dense in $R(\theta)$, then $Q(\theta)$ is an equivalence relation, and so $Q(\theta)=S(\theta)$. In particular $Q(\theta)=S(\theta)$ if $\theta$ is distal or if $\theta$ is open and $X$ has a $\theta$-distal point (a point $x$ not proximal to any point in the fiber of $\theta$ containing $x$ ).

TheOREM 1. Suppose $\theta$ is distal. Then $Q(\theta) \neq R(\theta)$.

Proof. Suppose not, suppose $Q(\theta)=R(\theta)$. Take a pseudometric, $d$, such that $d\left(x_{0}, x_{0}^{\prime}\right)=1$ for some $\left(x_{0}, x_{0}^{\prime}\right) \in R(\theta)$ and construct $K$ as above so that $\left(X_{K}, K\right)$ is minimal, $\phi_{K}\left(\theta^{-1}(y)\right)=\theta_{K}^{-1}\left(\psi_{K}(y)\right)$ for all $y$ in $Y$, and $\phi_{K} \times \phi_{K}(Q(\theta)) \subseteq Q\left(\theta_{K}\right)$. Then since $Q(\theta)=R(\theta)$, we have $Q\left(\theta_{K}\right)=R\left(\theta_{K}\right)$. Since $X_{K}$ is metric and $\theta_{K}$ is open, there are no $\theta_{K}$-distal points (see 2.1.5 of [V]). In particular take $u$ in $J_{T}$ and $u_{K}$ in $J_{K}$ such that $u u_{K}=u$ and take $x$ in $X$ with $x u=x$; and consider $\phi_{K}(x)$. Then there exists $x^{\prime}$ with $i\left(x, x^{\prime}\right) \in R(\theta), \phi_{K}(x) \neq \phi_{K}\left(x^{\prime}\right)$ and $\left(\phi_{K}(x), \phi_{K}\left(x^{\prime}\right)\right) \in$ $P\left(X_{K}, K\right)$. Note since $\theta$ is distal $x^{\prime} u=x^{\prime}$. There exists $p$ in $\beta K$ with $\phi_{K}(x p)=$ $\phi_{K}(x) p=\phi_{K}\left(x^{\prime}\right) p=\phi_{K}\left(x^{\prime} p\right)$; that is, $d_{K}\left(x p, x^{\prime} p\right)=0$. But $\left(x, x^{\prime}\right) u=\left(x, x^{\prime}\right)$, $\left(x, x^{\prime}\right) u_{K}=\left(x, x^{\prime}\right)$ is a $K$-almost periodic point and $\left(\operatorname{cls}\left(\left(x, x^{\prime}\right) K\right), K\right)$ is minimal. So $\left(x, x^{\prime}\right) \in \operatorname{cls}\left(\left(x p, x^{\prime} p\right) K\right)$, and clearly this gives a contradiction since $\phi_{K}(x) \neq$ $\phi_{K}\left(x^{\prime}\right)$ and so $d_{K}\left(x, x^{\prime}\right) \neq 0$. 
COROllary 2. If $\theta$ is distal, then $\theta: X \rightarrow Y$ is an I-extension.

Proposition 3. Suppose $X$ is a point-distal minimal flow, then $X$ is an HPI-flow. (Here we assume $T$ is discrete.)

Proof. Suppose not. Then there exist minimal flows and homomorphisms $\gamma: X_{\infty} \rightarrow X, \theta: X_{\infty} \rightarrow Y_{\infty}=Y$ where $\gamma$ is proximal, $Q(\theta)=R(\theta), \theta$ is not 1-to-1, $\theta$ is open, and $Y$ is HPI. Let $x_{0}$ be a distal point in $X$. Note we may assume $X_{\infty}=\operatorname{cls}\left(x_{0}, y_{0}\right) T \subseteq X \times Y$ for some $y_{0}$ in $Y$ and $\theta$ is the projection onto the second coordinate, by taking $X_{\infty}$ to be the unique minimal set in $X \times Y$. Let $d_{X}$ and $d_{Y}$ be pseudometrics in $X$ and $Y$, and let $d\left((x, y),\left(x^{\prime}, y^{\prime}\right)\right)=$ $\max \left\{d_{X}\left(x, x^{\prime}\right), d_{Y}\left(y, y^{\prime}\right)\right\}$ define a pseudometric on $X_{\infty}$. Note that if $\theta$ is proximal, then since it has a $\theta$-distal point $\left(x_{0}, y_{0}\right), \theta\left(y_{0}\right)$ is a singleton, and so since $\theta$ is open, $\boldsymbol{\theta}$ is not proximal.

Now as noted above we may take a subgroup $K$ of $T$ so that $\left(X_{\infty_{K}}, K\right)$ is a metric minimal flow, $\theta_{K}\left(\theta^{-1}(y)\right)=\theta_{K}^{-1}\left(\psi_{K}(y)\right), Q\left(\theta_{K}\right)=R\left(\theta_{K}\right)$, and $\theta_{K}$ is open, and not 1-to-1. Then by 2.1.5 of [V] no point in $X_{\infty_{K}}$ is a $\theta_{K}$-distal point.

Let $v \in J_{K}$ and consider the homomorphism of minimal flows $\left(\operatorname{cls}\left[\left(x_{0} v, y_{0} v\right) K\right], K\right) \subseteq\left(X_{\infty}, K\right) \rightarrow\left(X_{\infty_{K}}, K\right)$. Consider $\phi_{K}\left(x_{0} v, y_{0} v\right) \in X_{\infty_{K}}$; it is proximal to some other point $z$ in the same $\theta_{K}$-fiber. So there is some point $(x, y)$ in $\operatorname{cls}\left[\left(x_{0} v, y_{0} v\right) K\right]$ that is proximal to $\left(x_{0} v, y_{0} v\right)$ in $\left(\operatorname{cls}\left[\left(x_{0} v, y_{0} v\right) K\right], K\right)$ and has

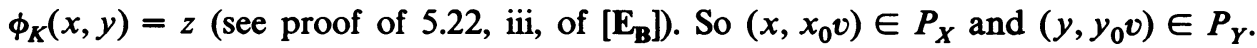
Now $\left(x_{0}, x_{0} v\right) v=\left(x_{0} v, x_{0} v v\right)=\left(x_{0} v, x_{0} v\right) \in \Delta$; so $\left(x_{0}, x_{0} v\right) \in P_{X}$ and $x_{0}=x_{0} v$ since $x_{0}$ is distal. So also $x=x_{0}$. Now since $\left(\phi_{K}\left(x_{0} v, y_{0} v\right), \phi_{K}(x, y)\right) \in R\left(\theta_{K}\right)$, we have $\left(\theta\left(x_{0} v, y_{0} v\right), \theta(x, y)\right) \in R\left(\psi_{K}\right)$; that is, $\left(y_{0} v, y\right) \in R\left(\psi_{K}\right)$, so $d_{K}^{*}\left(y_{0} v, y\right)=0$. Then there exists $x^{\prime}$ in $X$ so that $\left(x^{\prime}, y\right) \in X_{\infty}$ and $d_{K}\left(\left(x_{0}, y_{0} v\right),\left(x^{\prime}, y\right)\right)=0$. So $d_{X K}\left(x_{0}, x^{\prime}\right)=0$ and $d_{Y K}\left(y_{0} v, y\right)=0$, and therefore $d_{K}\left(\left(x_{0}, y_{0} v\right),\left(x_{0}, y\right)\right)=0$. Thus $\phi_{K}\left(x_{0}, y_{0} v\right)=\phi_{K}\left(x_{0}, y\right)=\phi_{K}(x, y)$, a contradiction.

\section{REFERENCES}

[AG] J. Auslander and S. Glasner, Distal and highly proximal extensions of minimal sets, Indiana Univ. Math. J. 26 (1977), 731-749.

[B] N. Bourbaki, General topology, Addison-Wesley, Reading, Mass., 1966.

[E] R. Ellis, The Furstenberg structure theorem, Pacific J. Math. 76 (1978), 345-349.

$\left[\mathbf{E}_{\mathrm{B}}\right] \ldots$, Lectures on topological dynamics, Benjamin, New York, 1969.

[G $\mathbf{G}_{\mathrm{B}}$ S. Glasner, Proximal flows, Lecture Notes in Math., vol. 517, Springer-Verlag, Berlin and New York, 1976.

[MN] D. McMahon and J. Nachman, An intrinsic characterization for PI-flows, Pacific J. Math. 89 (1980), 391-403.

[S] P. Shoenfeld, Highly proximal and generalized almost finite extensions of minimal sets, Pacific J. Math. 66 (1976), 265-280.

[V] W. A. Veech, Topological dynamics, Bull. Amer. Math. Soc. 83 (1977), 775-830.

Department of Mathematics, Arizona State University, Tempe, Arizona 85281

Department of Mathematics, Case Western Reserve University, Cleveland, Oho 44106 